\title{
Is Lipoprotein-Associated Phospholipase A2 a Link between Inflammation and Subclinical Atherosclerosis in Rheumatoid Arthritis?
}

\author{
Anna Södergren, ${ }^{1}$ Kjell Karp, ${ }^{2}$ Christine Bengtsson, ${ }^{1,3}$ Bozena Möller, ${ }^{4}$ \\ Solbritt Rantapää-Dahlqvist, ${ }^{1}$ and Solveig Wållberg-Jonsson ${ }^{1}$ \\ ${ }^{1}$ Department of Public Health and Clinical Medicine/Rheumatology, University of Umeå, 90187 Umeå, Sweden \\ ${ }^{2}$ Department of Surgical and Perioperative Sciences, University of Umeå, 90187 Umeå, Sweden \\ ${ }^{3}$ Department of Rheumatology, Östersund Hospital, 83127 Östersund, Sweden \\ ${ }^{4}$ Department of Rheumatology, Sunderby Hospital, 97189 Luleå, Sweden
}

Correspondence should be addressed to Anna Södergren; anna.sodergren@umu.se

Received 7 March 2015; Accepted 23 April 2015

Academic Editor: Giuseppe Biondi-Zoccai

Copyright (c) 2015 Anna Södergren et al. This is an open access article distributed under the Creative Commons Attribution License, which permits unrestricted use, distribution, and reproduction in any medium, provided the original work is properly cited.

Objective. Lipoprotein-associated phospholipase A2 (Lp-PLA2), a marker of vascular inflammation, is associated with cardiovascular disease. This prospective study of an inception cohort aimed to investigate whether the level of Lp-PLA2 is associated with subclinical atherosclerosis in patients with rheumatoid arthritis (RA). Methods. Patients from northern Sweden diagnosed with early RA were consecutively recruited into an ongoing prospective study. From these, all patients $\leq 60$ years $(n=71)$ were included for measurements of subclinical atherosclerosis at inclusion (T0) and five years later (T5). Forty age- and sex-matched controls were included. The patients were clinically assessed, SCORE, Reynolds Risk Score, and Larsen score were calculated, and blood samples were drawn from all individuals at T0 and T5. Results. There was no significant difference in the level of Lp-PLA2 between patients with RA and controls $(p>0.05)$. In simple linear regression models among patients with RA, Lp-PLA2 at T0 was significantly associated with intima media thickness (IMT) at T0 and T5, flow mediated dilation (FMD) at T0 and T5, ever smoking, male sex, HDL-cholesterol (inversely), non-HDL-cholesterol, SCORE, Reynolds Risk Score, and Larsen score $(p<0.05)$. Conclusion. In this cohort of patients with early RA, the concentration of Lp-PLA2 was associated with both subclinical atherosclerosis and disease severity.

\section{Introduction}

Patients with rheumatoid arthritis (RA) have increased atherosclerosis compared with the general population [14]. Atherosclerosis is now recognised as an inflammatory disease per se [5] and the two diseases, atherosclerosis and RA, are considered to share many similarities [6], albeit the link between them is, as yet, not evident.

Subclinical atherosclerosis precedes cardiovascular disease (CVD) and an increased intima media thickness (IMT), measured by ultrasonography, is regarded as an early indicator of a generalized atherosclerosis [7]. Several studies in the general population, as well as in patients with RA, have shown a relationship between an increased IMT and a future cardiovascular event [8-12]. We, and others, have previously shown that patients with established RA have a premature atherosclerosis as measured by an increased IMT of the common carotid artery (CCA) compared with controls [13, 14]. An even earlier sign of atherosclerosis, that is, endothelial dysfunction, indicated by an impaired flow mediated vascular dilation (FMD) of peripheral arteries, can also be measured using ultrasonography [15]. In the general population, FMD has been associated with other established risk factors for CVD and shown to be predictive of a future CV event [1517].

Lipoprotein-associated phospholipase A2 (Lp-PLA2), formerly also known as platelet-activating factor acetylhydrolase (PAF-AH), is an enzyme expressed, among others, 
by inflammatory cells in atherosclerotic plaques $[18,19]$. LpPLA2 hydrolyses phospholipids in low-density lipoprotein (LDL) to yield proinflammatory products such as oxidized free fatty acids. These proinflammatory products play a critical role in the endothelial chemotactic response by stimulating the expression of adhesion molecules and cytokines as well as recruiting inflammatory cells. Hence, Lp-PLA2 is suggested to be a useful and potent biomarker of the vascular inflammation involved in the pathophysiology of atherosclerosis [19-21].

In the circulation, Lp-PLA2 is carried bound mainly to LDL, and several epidemiological studies in the general population have shown a correlation between Lp-PLA2 levels and traditional cardiovascular risk factors [18, 22, 23]. In the general population, higher concentrations of Lp-PLA2 have also been shown to be associated with an increased risk of CVD [18]. Previous studies of patients with thalassemia, metabolic syndrome, or human immunodeficiency virus (HIV) infection, each diagnosis being characterised by an increased inflammation, have shown an association between Lp-PLA2 and subclinical atherosclerosis measured by IMT $[20,23,24]$. In populations without a known inflammation, however, the results are contradictory [21,25-27]. To the best of our knowledge there are no studies on the relationship between Lp-PLA2 and atherosclerosis in patients with RA.

In an ongoing prospective case-control study of patients with very early RA [28], we have found a significant increase in the subclinical atherosclerosis, measured by IMT and FMD, during the first five years of rheumatic disease [29]. In the present study, we hypothesized that vascular inflammation, reflected by the concentration of Lp-PLA2, contributes to the atherosclerotic disease in patients with RA. Thus, our primary aim was to investigate whether the level of Lp-PLA2 was associated with subclinical atherosclerosis at baseline (T0) or after the first five years following a diagnosis of RA (T5). A secondary aim was to identify markers of inflammation and traditional CVD risk factors associated with Lp-PLA2 and hence with a possible vascular inflammation preceding CVD.

\section{Material and Methods}

2.1. Patients and Controls. The present study is a part of a continuing structured programme on early RA for prospective analysis of CVD development in patients from northern Sweden using the nationwide Swedish Rheumatoid Arthritis Registry. All eligible patients with newly diagnosed RA (according to ACR criteria) [30] and symptomatic for no longer than 12 months are continuously enrolled into the register as soon as possible following diagnosis (T0). The inclusion criteria for the patients with RA and controls have previously been described in detail [28]. Five years after inclusion into the study (T5), 71 of the 79 patients with RA originally included were willing to participate in the followup study, and 40 of the original 44 controls were reassessed. The controls (one control for two patients except for in 13 cases one control per patient) were matched for age ( \pm 5 years) and sex. Only those individuals participating in the followup assessment, that is, T5, were included in this study. All individuals gave their written consent in accordance with the Declaration of Helsinki. The study was approved by the Regional Ethics Committee of Umeå University, Umeå, Sweden.

2.2. Physical Examination and Surveys. All patients were examined clinically at inclusion into the study and regularly thereafter at $3,6,12,18,24$, and 60 months. The number of swollen and tender joints (28-joint count) and the patient's global assessment were registered, and a disease activity score (DAS28), including the erythrocyte sedimentation rate (ESR), was calculated [31]. Posterior-anterior radiographs of the hands, wrists, and feet were obtained at baseline and after five years and were graded according to the Larsen score by two rheumatologists (EB and Solbritt Rantapää-Dahlqvist) [32]. All participants completed a survey on comorbidity. Any previous CVD events were verified by reference to medical records. Up to the five-year follow-up assessment, eight (11\%) of the patients with RA had suffered a CVD event ( 3 acute myocardial infarction, 3 stroke, and 2 thromboembolic event) whilst two (5\%) of the controls had suffered a CVD event (both coronary artery bypass graft surgery). Blood pressure was measured at the time of ultrasound measurements. Body mass index (BMI), European Systematic Coronary Risk Evaluation (SCORE) [33], and Reynolds Risk Score [34] were calculated at both $\mathrm{T} 0$ and $\mathrm{T} 5$. These compound measures of CVD risk factors estimate the risk of death due to a CVD event during the next 10 years. In addition to traditional CVD risk factors, the Reynolds Risk Score includes Creactive protein (CRP) concentrations. When calculating the Reynolds Risk Score, all patients were regarded as being nondiabetic due to a lack of information regarding levels of haemoglobin Alc for all individuals; this assumption may have resulted in an underestimation of the risk score.

2.3. Ultrasound Investigations. The patients were examined by ultrasound as soon as possible following diagnosis (at T0); the mean $( \pm \mathrm{SD})$ time after the primary symptom of RA was $16.2( \pm 6.6)$ months. The ultrasound investigations at the follow-up (T5) were performed 5 years after the initial examinations (mean and median being 60 months, with a range 5963, after the first examination). All ultrasonography examinations of patients with RA and controls were performed by the same experienced investigator (EL); the individuals were in a supine position in a quiet, temperature controlled room. A Sequoia 512 ultrasound system (Siemens (Acuson) Corp) was used with a 15L8 transducer for measurement of the brachial artery and an 8L5 transducer for carotid artery studies. All investigations were digitally stored for analyses to be performed by the single observer (EL; intraobserver variability for IMT $r=0.988)$. The protocol for these investigations has previously been described in detail [28].

2.4. Blood Sampling. In the present study all patients and controls donated a blood sample at the time of both ultrasound measurements, that is, at $\mathrm{T} 0$ and $\mathrm{T} 5$, and serum was stored at $-80^{\circ} \mathrm{C}$. After thawing, serum concentrations of Lp-PLA2 $(\mathrm{ng} / \mathrm{mL})$ were measured using an ELISA (R\&D 
TABLE 1: Measurement of Lp-PLA2, intima media thickness (IMT), flow mediated dilatation (FMD), traditional risk factors for cardiovascular disease (CVD), and disease activity in patients with early rheumatoid arthritis (RA) and in age- and sex-matched controls evaluated both at baseline (T0) and after 5 years (T5). Data are expressed as mean value (standard deviation).

\begin{tabular}{|c|c|c|c|c|}
\hline & \multicolumn{2}{|c|}{$\mathrm{RA}(n=71)$} & \multicolumn{2}{|c|}{ Controls $(n=40)$} \\
\hline & At T0 & At $\mathrm{T} 5$ & At T0 & At $\mathrm{T} 5$ \\
\hline Lp-PLA2, ng/mL & $144.0(41.7)$ & $154.6(38.9)^{*}$ & $132.0(37.8)$ & $148.1(41.7)^{* *}$ \\
\hline Intima media thickness, $\mathrm{mm}$ & $0.52(0.13)$ & $0.58(0.13)^{* * *}$ & $0.54(0.13)$ & $0.60(0.12)^{* * *}$ \\
\hline Endothelium dependent flow mediated vasodilatation, \% & $109.2(4.7)$ & $107.0(4.7)^{* * *}$ & $107.2(4.5)$ & $106.0(4.6)$ \\
\hline Systolic blood pressure, $\mathrm{mmHg}$ & $123.5(14.4)$ & $126.3(13.9)^{*}$ & $117.7(11.3)$ & $124.1(12.1)^{* * *}$ \\
\hline Cholesterol, $\mathrm{mmol} / \mathrm{L}$ & $5.5(0.9)$ & $5.3(1.0)$ & $5.3(1.1)$ & $5.6(1.1)$ \\
\hline $\mathrm{HDL}, \mathrm{mmol} / \mathrm{L}$ & $1.6(0.5)$ & $1.6(0.5)$ & $1.5(0.4)$ & $1.7(0.5)$ \\
\hline Non-HDL-cholesterol, $\mathrm{mmol} / \mathrm{L}$ & $3.9(0.9)$ & $3.7(1.0)$ & $3.9(1.1)$ & $3.9(1.1)$ \\
\hline Triglycerides, $\mathrm{mmol} / \mathrm{L}$ & $1.3(0.5)$ & $1.2(0.5)$ & $1.1(0.3)$ & $1.0(0.5)$ \\
\hline $\mathrm{BMI}, \mathrm{kg} / \mathrm{m}^{2}$ & $25.8(4.0)$ & $25.7(4.5)$ & $25.1(4.9)$ & $25.2(4.2)$ \\
\hline CRP, mg/L & $11.9(10.8)$ & $7.7(7.2)^{* *}$ & $\mathrm{n} / \mathrm{a}$ & $\mathrm{n} / \mathrm{a}$ \\
\hline DAS 28 & $3.5(1.4)$ & $3.1(1.5)^{\dagger}$ & $\mathrm{n} / \mathrm{a}$ & $\mathrm{n} / \mathrm{a}$ \\
\hline
\end{tabular}

${ }^{*} p$ value $<0.05 ;{ }^{* *} p$ value $<0.01 ;{ }^{* * *} p$ value $<0.0001 ;{ }^{\dagger} p$ value $=0.061 ;$ all values compared with $\mathrm{T} 0$.

Lp-PLA2: lipoprotein-associated phospholipase A2; HDL: high-density lipoproteins; BMI: body mass index; CRP: C-reactive protein; DAS28: disease activity score for 28 -joint count.

Systems, Abingdon, UK). Rheumatoid factor (RF; $67 \%$ of the patients were seropositive), CRP (mg/L), and erythrocyte sedimentation rate (ESR; $\mathrm{mm} / \mathrm{h}$ ) were measured according to routine methodology. Whenever several analyses of DAS28, CRP, or ESR were performed on any given individual, the assessment closest to the ultrasound measurement was used in any subsequent statistical analysis. Blood was also drawn after an overnight fast for analysis of blood lipids: cholesterol ( $\mathrm{mmol} / \mathrm{L})$, high-density lipoproteins (HDL; $\mathrm{mmol} / \mathrm{L})$, and triglycerides $(\mathrm{mmol} / \mathrm{L})$ using routine methods at each of the participating hospitals. Lp-PLA2 concentration results were available for 70 and 66 patients with RA at T0 and T5, respectively. Correspondingly, results for Lp-PLA2 were available for 38 and 40 controls at T0 and T5, respectively.

2.5. Statistics. Comparisons over time within the RA patient group and within the control group were performed using the Wilcoxon paired test. Simple and multiple linear regression analyses were used to identify variables associated with LpPLA2. Results from simple linear regression (variables with $p<0.05$ ), together with clinical assumptions (variables with $p>0.05$ ), determined which covariates were included in the multiple linear regression models. Differences in variables between patients with RA and matched controls were analysed using simple conditional logistic regression analyses. Occasional missing values, due to missing information, were regarded as random. Non-HDL-cholesterol was calculated as total cholesterol minus HDL-cholesterol. Based on previously published data [20], calculations showed that a sample size of 71 would render $99 \%$ power to detect a correlation between IMT and Lp-PLA2 with a correlation coefficient of 0.46. $p$ values $<0.05$ were considered statistically significant. All calculations were made using SPSS 20.0 (SPSS Inc., Chicago, USA).

\section{Results}

For this study, 71 patients with RA (61 (86\%) women) and 40 controls (32 (80\%) women) were included. The mean age (SD) of the patients with RA was 51.5 (10.7) years and 48.1 (10.9) years for the controls. Among the patients with RA, 35 (58\%) declared themselves to ever being a smoker, corresponding with 14 (39\%) among the controls. Six (9\%) patients with RA and $5(12 \%)$ controls had ever used statins.

The concentration of Lp-PLA2 increased significantly during the 5-year follow-up period, both for the patients with RA and the controls (Table 1). At both time points the concentrations of Lp-PLA2 were numerically higher in patients with RA compared with controls ( $p$ values $>0.05$ ) (Table 1).

Among the patients with RA, the concentration of LpPLA2 at T0 was significantly associated with IMT as well as with FMD at both baseline and follow-up (Table 2). After adjustment for sex and age, Lp-PLA2 was still significantly associated with IMT at T0 and T5 (Table 2). At T0, the LpPLA2 concentration was also significantly associated with non-HDL-cholesterol, HDL (inversely), diastolic blood pressure, smoking, SCORE, and Reynolds Risk Score as well as the Larsen score (Table 2). Adjustment for disease activity, measured by DAS28 at T0, did not change significantly the association between the Lp-PLA2 levels at T0 and disease severity measured by the Larsen score at T0 (Table 3 ).

At T5 Lp-PLA2 was significantly associated with nonHDL-cholesterol, HDL (inversely), cholesterol, BMI, and Reynolds Risk Score among the patients with RA (Table 4). There were no associations between Lp-PLA2 and any of the measures of disease activity, that is, CRP, ESR, and DAS28, at either T0 or T5 (data not shown). Furthermore, there was no significant association between Lp-PLA2 concentration and the Larsen score at T5, nor were there any significant 
TABLE 2: Results of simple regression models among the 71 patients with early RA with the concentration of Lp-PLA2 at T0 as the dependent variable.

\begin{tabular}{lccc}
\hline & \multicolumn{3}{c}{ Lp-PLA2 at T0 } \\
& $\beta$ & $95 \%$ CI & $p$ value \\
\hline IMT T0 $(n=70)$ & $9.7 / \mathrm{mm}$ & $2.1 ; 17.2$ & $0.013^{\dagger}$ \\
IMT T5 $(n=70)$ & $8.8 / \mathrm{mm}$ & $1.4 ; 16.2$ & $0.02^{\dagger}$ \\
FMD T0 $(n=70)$ & $-2.4 / \%$ & $-4.5 ;-0.4$ & 0.02 \\
FMD T5 $(n=70)$ & $-2.5 / \%$ & $-4.6 ;-0.5$ & 0.02 \\
Non-HDL-cholesterol & $16.9 / \mathrm{mmolL}^{-1}$ & $5.8 ; 28.0$ & 0.004 \\
T0 $(n=54)$ & $-22.6 / \mathrm{mmolL}^{-1}$ & $-42.0 ;-3.1$ & 0.02 \\
HDL T0 $(n=55)$ & $1.3 / \mathrm{mmHg}$ & $0.1 ; 2.5$ & 0.04 \\
Diastolic blood pressure & & $0.08 ; 1.4$ & 0.03 \\
T0 $(n=66)$ & $0.8 / \mathrm{year}$ & $3.3 ; 19.5$ & 0.007 \\
Ever smoking $(n=70)$ & $11.4 / \mathrm{unit}$ & $1.5 ; 8.8$ & 0.007 \\
SCORE T0 $(n=53)$ & $5.2 / \mathrm{unit}$ & $0.013 ; 5.90$ & 0.05 \\
Reynolds Risk Score T0 & & & \\
$(n=38)$ & $2.9 / \mathrm{unit}$ & &
\end{tabular}

${ }^{\dagger}$ Still significant after adjustment for sex and age.

RA: rheumatoid arthritis; Lp-PLA2: lipoprotein-associated phospholipase A2; IMT: intima media thickness; FMD: flow mediated dilation; HDL: highdensity lipoproteins.

TABLE 3: Multiple regression models among 71 patients with early RA with the concentration of Lp-PLA2 at T0 as dependent variable.

\begin{tabular}{lccc}
\hline & \multicolumn{3}{c}{ Lp-PLA2 at T0 } \\
& $\beta$ & $95 \%$ CI & $p$ value \\
\hline Larsen score T0 & 2.8/unit & $-0.3 ; 5.8$ & 0.06 \\
DAS28 T0 & $-4.6 /$ unit & $-2.0 ; 0.3$ & 0.03 \\
\hline
\end{tabular}

RA: rheumatoid arthritis; Lp-PLA2: lipoprotein-associated phospholipase A2; DAS28: disease activity score for 28 -joint count.

associations between Lp-PLA2 and age, or any medication either at T0 or at T5 (data not shown).

Among the controls, Lp-PLA2 at T0 was significantly associated with IMT at T0 $(\beta 3.2, p=0.05)$ and LpPLA2 at T5 was significantly associated with several variables measured at T0: IMT ( $\beta 3.9, p<0.05)$, cholesterol $(\beta$ 6.1, $p<0.001)$, triglycerides $(\beta 3.3, p<0.05)$, diastolic blood pressure $(\beta 4.1, p=0.01)$, age $(\beta 5.2, p<0.001)$, and SCORE $(\beta$ 4.1, $p<0.05)$. Among the same individuals Lp-PLA2 at T5 was significantly associated with IMT $(\beta 3.3, p<0.05)$ and cholesterol $(\beta 5.8, p<0.001)$ at T5. After adjustment for sex and age no significant association between Lp-PLA2 and IMT was found among the controls, neither at T0 nor at T5 (data not shown).

\section{Discussion}

In this study, the serum concentration of Lp-PLA2 was associated with measures both of subclinical atherosclerosis over time and of disease severity at disease onset in patients with early RA.
TABLE 4: Results of simple regression models among 71 patients with early RA with the concentration of Lp-PLA2 at T5 as the dependent variable.

\begin{tabular}{lccc}
\hline & \multicolumn{3}{c}{ Lp-PLA2 at T5 } \\
& $\beta$ & $95 \%$ CI & $p$ value \\
\hline IMT T5 $(n=66)$ & $4.4 / \mathrm{mm}$ & $-2.7 ; 11.5$ & 0.22 \\
FMD T5 $(n=66)$ & $-1.7 / \%$ & $-3.8 ; 0.4$ & 0.10 \\
Non-HDL-cholesterol & $19.0 / \mathrm{mmolL}^{-1}$ & $10.5 ; 27.5$ & 0.001 \\
T5 $(n=61)$ & $-31.8 / \mathrm{mmolL}^{-1}$ & $-50.3 ;-13.3$ & 0.001 \\
HDL T5 $(n=61)$ & $11.5 / \mathrm{mmolL}^{-1}$ & $2.1 ; 20.8$ & 0.02 \\
Cholesterol T5 $(n=61)$ & $0.4 /$ year & $-0.3 ; 1.1$ & 0.26 \\
Ever smoking $(n=66)$ & $2.5 /$ unit & $0.3 ; 4.7$ & 0.03 \\
BMI T5 $(n=66)$ & $3.6 /$ unit & $-2.0 ; 9.2$ & 0.21 \\
SCORE T5 $(n=61)$ & $2.8 /$ unit $_{\text {Reynolds Risk Score T5 }}$ & $-0.2 ; 5.7$ & 0.06 \\
$(n=39)$ & & & \\
\hline
\end{tabular}

RA: rheumatoid arthritis; Lp-PLA2: lipoprotein-associated phospholipase A2; IMT: intima media thickness; FMD: flow mediated dilation; HDL: highdensity lipoproteins; BMI: body mass index.

From previously published reports, it is evident that patients with RA have an increased development of atherosclerosis compared with the general population, with different underlying causes being proposed to explain this observation $[3,4,6,13]$. A strong theory to date is that the inflammatory load among the patients with RA affects the arteries and gives rise to a subclinical vascular inflammation. An increased level of Lp-PLA2 among patients with RA was shown several years ago, when it was presented as a marker of disease activity among such patients [35, 36]. However, more recently published studies on other inflammatory diseases, as well as on the general population, suggest that Lp-PLA2 cannot be regarded as a marker of a systemic inflammation but as a mere biomarker of atherosclerosis [19]. With this background in mind, we measured the concentration of Lp-PLA2 as a marker of vascular inflammation. However no significant difference in the levels of Lp-PLA2 in patients with RA and controls was found, neither early in the disease nor after 5 years, albeit the concentrations of Lp-PAL2 were numerically higher among the patients with RA at all time points.

It is now recognized that atherosclerosis is the result of an inflammatory process in the vessel wall [5], and early atherosclerosis can be identified as an endothelial dysfunction by FMD or arterial wall thickening by IMT. The Lp-PLA2 concentration at inclusion of patients with early RA into this study, as well as at the five-year followup assessment, was found to be associated with both measurements of early atherosclerosis. Lp-PLA2 is regarded as a highly specific biomarker for vascular inflammation and burden of atherosclerosis [19]. The correlation between this biomarker and atherosclerosis is well studied in the general population, as well as in other inflammatory diseases; however there are also contradictory results [20, 21, 23-27]. After five years following a diagnosis of RA, the levels of Lp-PLA2 found at inclusion could still explain the extent 
of atherosclerosis measured by IMT and FMD in patients with RA. There are, to the best of our knowledge, only a few prospective studies on Lp-PLA2 and the development of subclinical atherosclerosis and none regarding patients with an inflammatory disease. Two studies on patients with diabetes mellitus found measurements of Lp-PLA2 to be associated with the progression of atherosclerosis over time $[37,38]$ and Liu et al. verified these results in the general population [27]. In this study a similar result was found, with an association between Lp-PLA2 and prospectively registered measures of atherosclerosis in patients with early RA.

In previous studies involving this cohort of patients, the extent of atherosclerosis was associated with traditional cardiovascular risk factors $[28,29]$. In the present study we found, consistent with other published studies, the levels of Lp-PLA2 to be associated with several traditional risk factors $[18,22,23]$. Moreover, the compound measure of CVD risk that included inflammation, that is, the Reynolds Risk Score, was strongly associated with Lp-PLA2 at both T0 and T5. The Lp-PLA2 molecule is carried in the circulation mainly bound to LDL $[18,19]$ and, as was to be expected, the level of Lp-PLA2 was found to be evidently associated with the concentrations of blood lipids. Non-HDL-cholesterol, in some regards a better measurement of the risk of CVD than LDL [39], was strongly associated with Lp-PLA2 both at T0 and at $\mathrm{T} 5$.

Radiological progression is a measurement of disease severity over time in patients with RA. We found a significant relationship between Lp-PLA2 concentration and the Larsen score at the time of diagnosis of RA. In these patients, the same inflammatory process that leads to joint damage may also affect the vascular walls causing a vascular inflammation, as reflected by elevated concentrations of Lp-PLA2. This association was not altered significantly by adjustment for disease activity at inclusion, again indicating that Lp-PLA2 is not just a marker of disease activity. In a multiple regression model, both IMT and Larsen score at inclusion were significantly associated with the concentration of Lp-PLA2, indicating that the processes leading to joint damage and vascular damage are, in some part, interlinked.

The main strength of the present study is the prospective design from the onset of disease. In northern Sweden almost all individuals newly diagnosed with RA are included in a structured follow-up programme. Of these patients, all of those aged $\leq 60$ years were invited to participate in this study within 12 months of their diagnosis. Data on biomarkers and traditional CVD risk factors, as well as variables related to the RA disease, were collected from the onset of disease and then continuously during the five years of follow-up. Another strength of this study is that the same person (KE) undertook all of the laboratory-based analyses and that Lp-PLA2 in samples collected at both time points (i.e., T0 and T5) was measured simultaneously. Furthermore, all ultrasound measurements at both time points, and their analysis, were undertaken by the same person (EL).

Conversely, a limitation of this study is that it is strictly observational; in other words, no influence could be made on medications and other variables observed. Another limitation is the number of control subjects; however this study was directed primarily at studying the serum concentrations of Lp-PLA2 among the patients with RA. Another limitation is the lack of data on LDL-cholesterol levels. However, non-HDL-cholesterol was calculated, based on findings reported in some studies that it is more strongly associated with a risk of CVD than LDL-cholesterol [39]. The relationship between Lp-PLA2 and LDL-cholesterol must be regarded as well studied $[18,22]$. Furthermore, we were not able to explore the association between Lp-PLA2 and CVD since there were too few CV events during the follow-up. Still, this study is the first of its kind, and further studies, including a follow-up of the individuals in the present study, will be able to clarify this association.

\section{Conclusions}

In this study, the level of Lp-PLA2 among patients with RA was associated with subclinical atherosclerosis, prospectively measured by IMT and FMD. Among these patients with early RA, this biomarker of vascular inflammation was also associated with Larsen score, indicating that over time the deleterious disease process may also affect the vascular walls. Taken together, our findings indicate a continuous vascular inflammation among patients with RA possibly leading to the development of atherosclerosis and hence to CVD. This possibility adds to the knowledge of the mechanisms responsible for the observed increased risk of CVD among patients with RA.

\section{Conflict of Interests}

None of the authors declare any potential conflict of interests.

\section{Acknowledgments}

The authors thank Ms. Elisabet Lundström at the Department of Surgical and Perioperative Sciences, University of Umeå, who carried out all of the ultrasound measurements. They also thank Ms. Gun-Britt Johansson, Ms. Ann-Chatrin Kallin, and Ms. Sonja Odeblom at the Department for Rheumatology, University Hospital, Umeå, for their excellent help with collection of patient data, and Ms. Kristina Eriksson, Department of Medicine, University Hospital, Umeå, for excellent measurement of the biomarkers. Furthermore, they thank Ms. Ewa Berglin, M.D., Ph.D., at the Department for Rheumatology, University Hospital, Umeå, for grading the radiographs according to the Larsen score. This work was supported by grants from the Swedish Research Council (Grant no. K 2007-52X-20307-01-3); the Swedish Rheumatism Association; the Swedish Rheumatism Association in the Västerbotten County; Visare Norr, Norrlandstingens Regionförbund (Northern County Councils); the Swedish Heart-Lung Foundation; the King Gustaf V's 80-Year Fund, Sweden; and the Swedish Society for Medical Research (SSMF). 


\section{References}

[1] S. Wållberg-Jonsson, H. Johansson, M.-L. Öhman, and S. Rantapää-Dahlqvist, "Extent of inflammation predicts cardiovascular disease and overall mortality in seropositive rheumatoid arthritis. A retrospective cohort study from disease onset," Journal of Rheumatology, vol. 26, no. 12, pp. 2562-2571, 1999.

[2] I. del Rincón, G. L. Freeman, R. W. Haas, D. H. O’Leary, and A. Escalante, "Relative contribution of cardiovascular risk factors and rheumatoid arthritis clinical manifestations to atherosclerosis," Arthritis and Rheumatism, vol. 52, no. 11, pp. 3413-3423, 2005.

[3] P. H. Dessein, B. I. Joffe, M. G. Veller et al., "Traditional and nontraditional cardiovascular risk factors are associated with atherosclerosis in rheumatoid arthritis," Journal of Rheumatology, vol. 32, no. 3, pp. 435-442, 2005.

[4] J. T. Giles, W. S. Post, R. S. Blumenthal et al., "Longitudinal predictors of progression of carotid atherosclerosis in rheumatoid arthritis," Arthritis \& Rheumatism, vol. 63, no. 11, pp. 3216-3225, 2011.

[5] G. K. Hansson, A. K. Robertson, and C. Soderberg-Naucler, "Inflammation and atherosclerosis," Annual Review of Pathology, vol. 1, pp. 297-329, 2006.

[6] D. F. van Breukelen-van der Stoep, B. Klop, D. van Zeben, J. M. W. Hazes, and M. C. Cabezas, "Cardiovascular risk in rheumatoid arthritis: how to lower the risk?" Atherosclerosis, vol. 231, no. 1, pp. 163-172, 2013.

[7] F. Molinari, G. Zeng, and J. S. Suri, "A state of the art review on intima-media thickness (IMT) measurement and wall segmentation techniques for carotid ultrasound," Computer Methods and Programs in Biomedicine, vol. 100, no. 3, pp. 201-221, 2010.

[8] D. H. O'Leary and M. L. Bots, "Imaging of atherosclerosis: carotid intima-media thickness," European Heart Journal, vol. 31, no. 14, pp. 1682-1689, 2010.

[9] M. W. Lorenz, H. S. Markus, M. L. Bots, M. Rosvall, and M. Sitzer, "Prediction of clinical cardiovascular events with carotid intima-media thickness: a systematic review and metaanalysis," Circulation, vol. 115, no. 4, pp. 459-467, 2007.

[10] J. F. Polak, M. Szklo, R. A. Kronmal et al., "The value of carotid artery plaque and intima-media thickness for incident cardiovascular disease: the multi-ethnic study of atherosclerosis," Journal of the American Heart Association, vol. 2, no. 2, Article ID e000087, 2013.

[11] C. Gonzalez-Juanatey, J. Llorca, J. Martin, and M. A. GonzalezGay, "Carotid intima-media thickness predicts the development of cardiovascular events in patients with rheumatoid arthritis," Seminars in Arthritis \& Rheumatism, vol. 38, no. 5, pp. 366-371, 2009.

[12] M. R. Evans, A. Escalante, D. F. Battafarano, G. L. Freeman, D. H. O'Leary, and I. del Rincõn, "Carotid atherosclerosis predicts incident acute coronary syndromes in rheumatoid arthritis," Arthritis and Rheumatism, vol. 63, no. 5, pp. 1211-1220, 2011.

[13] S. Wållberg-Jonsson, C. Backman, O. Johnson et al., "Increased prevalence of atherosclerosis in patients with medium term rheumatoid arthritis," The Journal of Rheumatology, vol. 28, no. 12, pp. 2597-2602, 2001.

[14] A. M. van Sijl, M. J. Peters, D. K. Knol et al., "Carotid intima media thickness in rheumatoid arthritis as compared to control subjects: a meta-analysis," Seminars in Arthritis and Rheumatism, vol. 40, no. 5, pp. 389-397, 2011.
[15] M. Charakida, S. Masi, T. F. Lüscher, J. J. P. Kastelein, and J. E. Deanfield, "Assessment of atherosclerosis: the role of flowmediated dilatation," European Heart Journal, vol. 31, no. 23, pp. 2854-2861, 2010.

[16] D. J. Green, H. Jones, D. Thijssen, N. T. Cable, and G. Atkinson, "Flow-mediated dilation and cardiovascular event prediction: does nitric oxide matter?” Hypertension, vol. 57, no. 3, pp. 363369, 2011.

[17] M. Shechter, A. Shechter, N. Koren-Morag, M. S. Feinberg, and L. Hiersch, "Usefulness of brachial artery flow-mediated dilation to predict long-term cardiovascular events in subjects without heart disease," American Journal of Cardiology, vol. 113, no. 1, pp. 162-167, 2014.

[18] A. Thompson, P. Gao, L. Orfei et al., "Lipoprotein-associated phospholipase $\mathrm{A}_{2}$ and risk of coronary disease, stroke, and mortality: collaborative analysis of 32 prospective studies," The Lancet, vol. 375, no. 9725, pp. 1536-1544, 2010.

[19] A. Cai, D. Zheng, R. Qiu, W. Mai, and Y. Zhou, "Lipoproteinassociated phospholipase A2 (Lp-PLA-2): a novel and promising biomarker for cardiovascular risks assessment," Disease Markers, vol. 34, no. 5, pp. 323-331, 2013.

[20] A. D. Tselepis, G. Hahalis, C. C. Tellis et al., "Plasma levels of lipoprotein-associated phospholipase $\mathrm{A}_{2}$ are increased in patients with $\beta$-thalassemia," Journal of Lipid Research, vol. 51, no. 11, pp. 3331-3341, 2010.

[21] P. K. Garg, R. L. McClelland, N. S. Jenny et al., "Association of lipoprotein-associated phospholipase $\mathrm{A}_{2}$ and endothelial function in the Multi-Ethnic Study of Atherosclerosis (MESA)," Vascular Medicine, vol. 16, no. 4, pp. 247-252, 2011.

[22] O. Vittos, B. Toana, A. Vittos, and E. Moldoveanu, "Lipoproteinassociated phospholipase A2 (Lp-PLA2): a review of its role and significance as a cardiovascular biomarker," Biomarkers, vol. 17, no. 4, pp. 289-302, 2012.

[23] H.-P. Gong, Y.-M. Du, L.-N. Zhong et al., "Plasma lipoproteinassociated phospholipase A2 in patients with metabolic syndrome and carotid atherosclerosis," Lipids in Health and Disease, vol. 10, article 13, 2011.

[24] A. R. Eckard, C. Longenecker, Y. Jiang et al., "Lipoproteinassociated phospholipase $\mathrm{A}_{2}$ and cardiovascular disease risk in HIV infection," HIV Medicine, vol. 15, no. 9, pp. 537-546, 2014.

[25] D. N. Kiortsis, S. Tsouli, E. S. Lourida et al., "Lack of association between carotid intima-media thickness and PAFacetylhydrolase mass and activity in patients with primary hyperlipidemia," Angiology, vol. 56, no. 4, pp. 451-458, 2005.

[26] S. Campo, M. A. Sardo, A. Bitto et al., "Platelet-activating factor acetylhydrolase is not associated with carotid intima-media thickness in hypercholesterolemic sicilian individuals," Clinical Chemistry, vol. 50, no. 11, pp. 2077-2082, 2004.

[27] J. Liu, W. Wang, Y. Qi et al., "Association between the lipoprotein-associated phospholipase $\mathrm{A}_{2}$ activity and the progression of subclinical atherosclerosis," Journal of Atherosclerosis and Thrombosis, vol. 21, pp. 532-542, 2014.

[28] A. Södergren, K. Karp, K. Boman et al., "Atherosclerosis in early rheumatoid arthritis: very early endothelial activation and rapid progression of intima media thickness," Arthritis Research \& Therapy, vol. 12, no. 4, article R158, 2010.

[29] A. Södergren, K. Karp, C. Bengtsson, B. Möller, S. RantapääDahlqvist, and S. Wållberg-Jonsson, "The extent of subclinical atherosclerosis is partially predicted by the inflammatory load: a prospective study over 5 years in patients with rheumatoid arthritis and matched controls," The Journal of Rheumatology, 2015. 
[30] F. C. Arnett, S. M. Edworthy, D. A. Bloch et al., "The American Rheumatism Association 1987 revised criteria for the classification of rheumatoid arthritis," Arthritis \& Rheumatism, vol. 31, no. 3, pp. 315-324, 1988.

[31] M. L. L. Prevoo, M. A. van 'T Hof, H. H. Kuper, M. A. van Leeuwen, L. B. A. van De Putte, and P. L. C. M. van Riel, "Modified disease activity scores that include twenty-eight-joint counts: development and validation in a prospective longitudinal study of patients with rheumatoid arthritis," Arthritis and Rheumatism, vol. 38, no. 1, pp. 44-48, 1995.

[32] A. Larsen, "How to apply larsen score in evaluating radiographs of rheumatoid arthritis in longterm studies?" Journal of Rheumatology, vol. 22, no. 10, pp. 1974-1975, 1995.

[33] R. M. Conroy, K. Pyörälä, A. P. Fitzgerald et al., "Estimation of ten-year risk of fatal cardiovascular disease in Europe: the SCORE project," European Heart Journal, vol. 24, no. 11, pp. 9871003, 2003.

[34] P. M. Ridker, J. E. Buring, N. Rifai, and N. R. Cook, "Development and validation of improved algorithms for the assessment of global cardiovascular risk in women: the Reynolds Risk Score," Journal of the American Medical Association, vol. 297, no. 6, pp. 611-619, 2007.

[35] A. Dulioust, P. Hilliquin, C.-J. Menkes, J. Benveniste, and B. Arnoux, "Paf-acether acetylhydrolase activity is increased in patients with rheumatic diseases," Scandinavian Journal of Rheumatology, vol. 21, no. 4, pp. 161-164, 1992.

[36] P. Hilliquin, A. Dulioust, C. Gregoir, A. Arnoux, and C. J. Menkes, "Production of PAF-acether by synovial fluid neutrophils in rheumatoid arthritis," Inflammation Research, vol. 44, no. 8, pp. 313-316, 1995.

[37] G. L. Kinney, J. K. Snell-Bergeon, D. M. Maahs et al., "Lipoprotein-associated phospholipase $\mathrm{A}_{2}$ activity predicts progression of subclinical coronary atherosclerosis," Diabetes Technology \& Therapeutics, vol. 13, no. 3, pp. 381-387, 2011.

[38] A. Saremi, T. E. Moritz, R. J. Anderson, C. Abraira, W. C. Duckworth, and P. D. Reaven, "Rates and determinants of coronary and abdominal aortic artery calcium progression in the Veterans Affairs Diabetes Trial (VADT)," Diabetes Care, vol. 33, no. 12, pp. 2642-2647, 2010.

[39] S. M. Boekholdt, B. J. Arsenault, S. Mora et al., "Association of LDL cholesterol, non-HDL cholesterol, and apolipoprotein B levels with risk of cardiovascular events among patients treated with statins: a meta-analysis," The Journal of the American Medical Association, vol. 307, no. 12, pp. 1302-1309, 2012. 


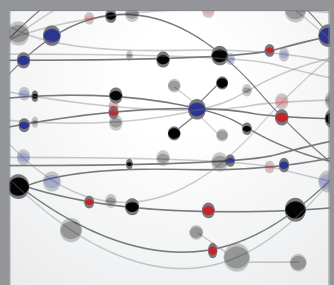

The Scientific World Journal
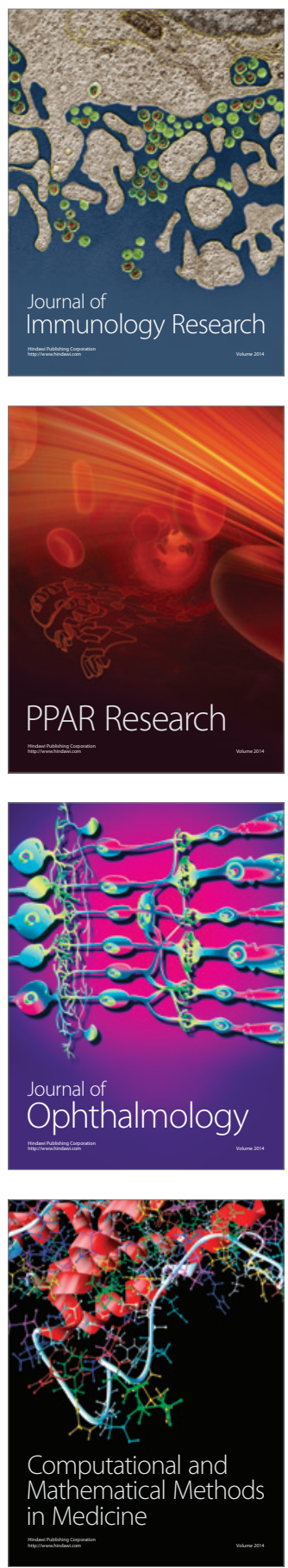

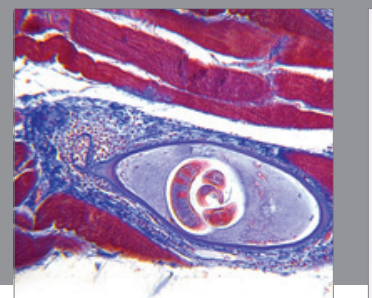

Gastroenterology

Research and Practice
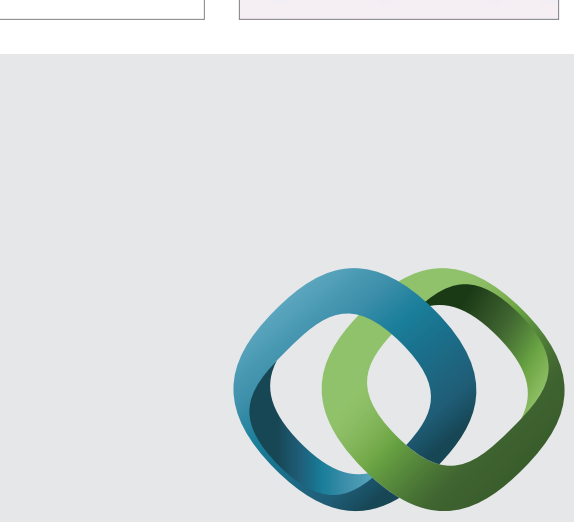

\section{Hindawi}

Submit your manuscripts at

http://www.hindawi.com
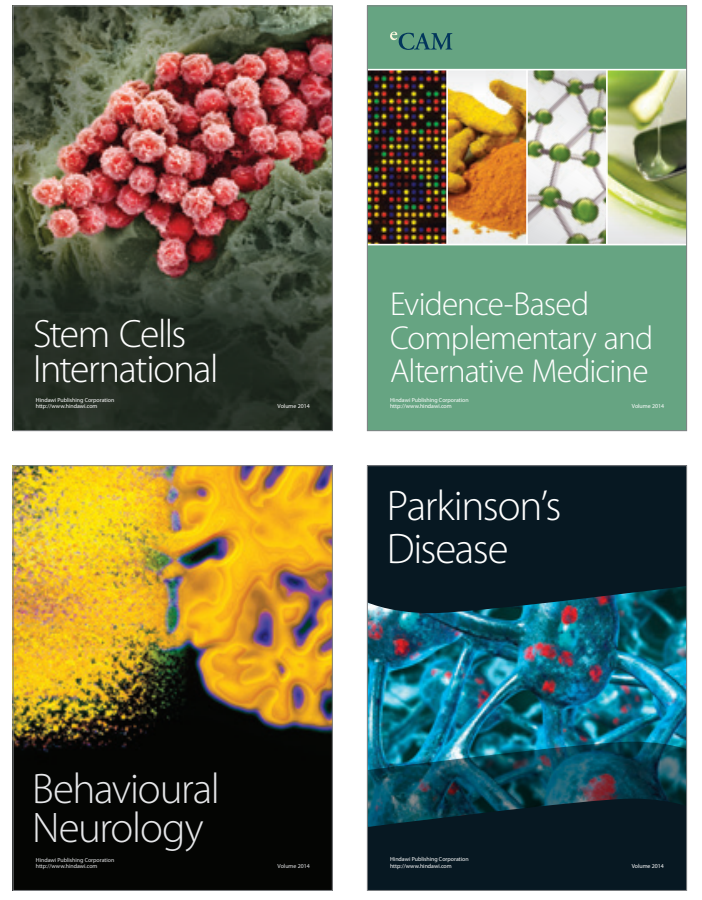
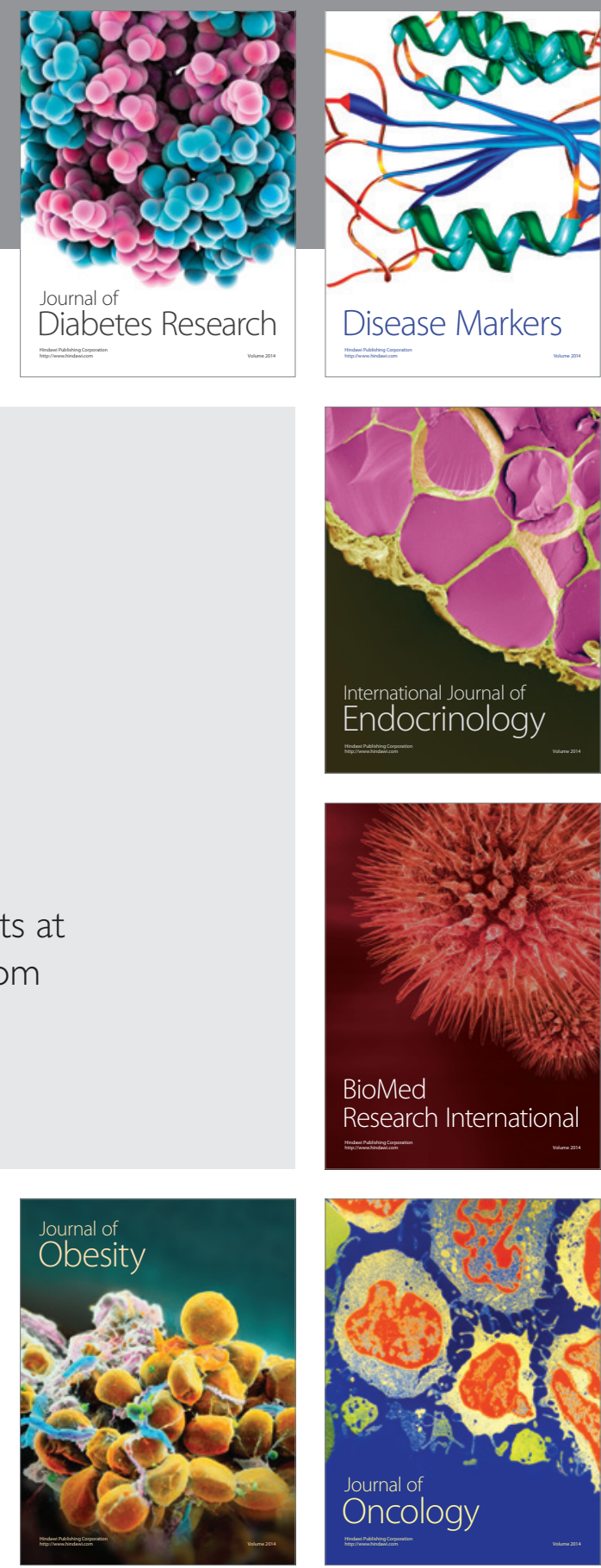

Disease Markers
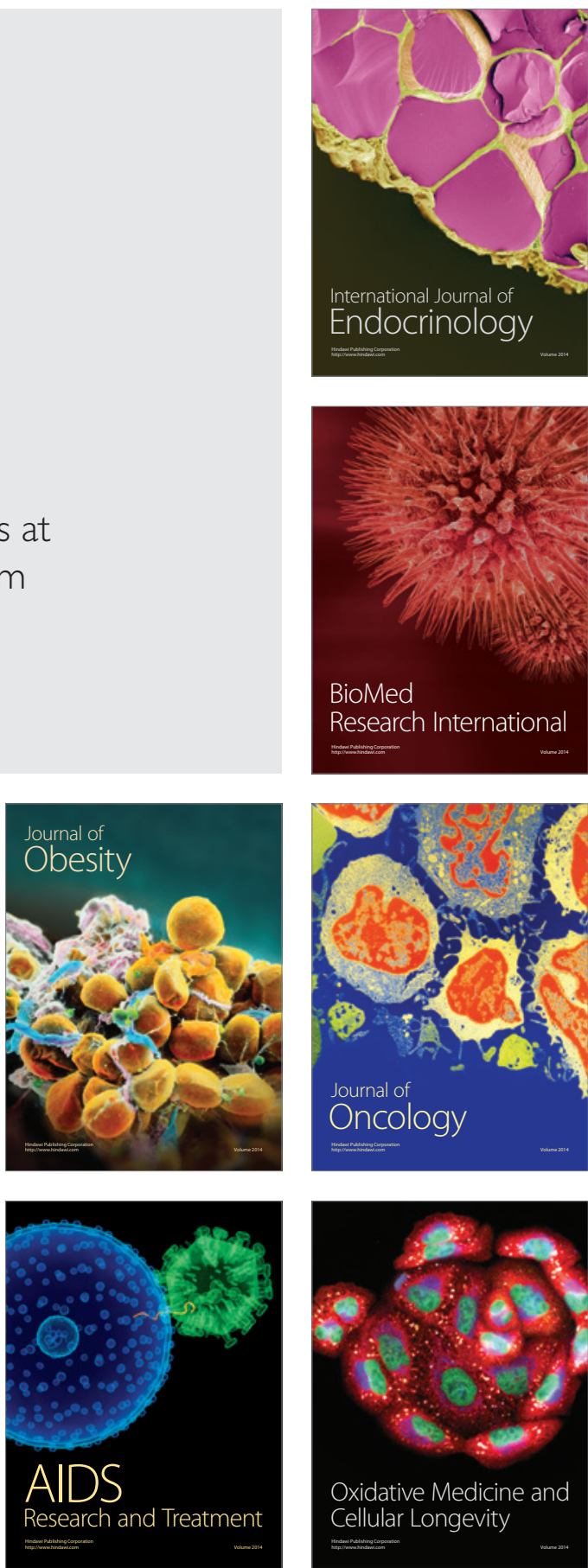